\title{
Efficacy and Safety of Subcutaneous Anti-Tumor Necrosis Factor-Alpha Agents, Etanercept and Adalimumab, in Elderly Patients Affected by Psoriasis and Psoriatic Arthritis: An Observational Long-Term Study
}

\author{
Maria Esposito ${ }^{a} \quad$ Alessandro Giunta ${ }^{a} \quad$ Annamaria Mazzotta ${ }^{a}$ Arianna Zangrilli ${ }^{a}$ \\ Graziella Babino $^{a}$ Mauro Bavetta ${ }^{a}$ Roberto Perricone ${ }^{b}$ Sergio Chimenti ${ }^{a}$ \\ Maria Sole Chimentib \\ Departments of a Dermatology and ${ }^{b}$ Rheumatology, University of Rome 'Tor Vergata', Rome, Italy
}

\section{Key Words}

Adalimumab · Anti-TNF-alpha • Elderly • Etanercept •

Psoriasis

\begin{abstract}
Background: In elderly patients the management of psoriasis is challenging due to contraindications and a higher risk of side effects. Objective: Our retrospective study aimed to evaluate the long-term efficacy and safety profile of subcutaneous anti-tumor necrosis factor (anti-TNF) agents in elderly psoriatic patients. Methods: The study included 89 patients (aged $\geq 65$ years) with plaque-type psoriasis and psoriatic arthritis treated with the subcutaneous anti-TNF- $\alpha$ agents etanercept or adalimumab as monotherapy for a long-term continuous period. Results: Efficacy results were consistent and stable over long-term observation, as expressed by mean Psoriasis Area and Severity Index (PASI) score variation, percentage of patients achieving PASI50 and PASI75 and by the improvement of articular indices, pain visual analogue scale (Pain-VAS) and 44-Joint Disease Activity Score (DAS44-ESR). The proportion of patients achieving
\end{abstract}

PASI50 was 91.80 and $82.14 \%$ at week 156 with etanercept and adalimumab treatment, respectively, while the proportion of patients achieving PASI75 was 83.61 and $71.43 \%$ at week 156 when treated with etanercept and adalimumab, respectively. The mean DAS44-ESR score decreased from 5.80 to 0.89 and from 3.43 to 1.44 at week 156 and the mean Pain-VAS score decreased from 75.10 to 3.15 and from 71.30 to 18.26 at week 156 with etanercept and adalimumab treatment, respectively. Both treatment adherence and safety profile were good. Conclusions: Our study demonstrates that subcutaneous anti-TNF- $\alpha$ agents are appropriate in the long-term management of elderly patients.

Copyright $\odot 2012$ S. Karger AG, Basel

\section{Introduction}

Psoriasis (Pso) and psoriatic arthritis (PsA) are chronic and disabling inflammatory diseases, characterized by an unpredictable course involving relapses. Pso affects $2-3 \%$ of the world's population with a negative impact on the quality of life [1-3]. Long-term management of these

\section{KARGER}

E-Mail karger@karger.com

www.karger.com/drm
(C) 2012 S. Karger AG, Basel

$1018-8665 / 12 / 2254-0312 \$ 38.00 / 0$ 
diseases with traditional systemic therapies is limited due to a lack of efficacy and potential organ toxicity. However, long-term management of Pso has significantly improved in recent decades following the introduction of targeted biological agents [4]. Several trials have shown excellent clinical results with the anti-tumor necrosis factor- $\alpha$ (TNF- $\alpha)$ agents, etanercept $[5,6]$, infliximab [7] and adalimumab $[8,9]$, which have been demonstrated to be effective in different aspects of the disease, including skin lesions, joint manifestations, enthesitis, and dactylitis, leading to a significant improvement in radiographic progression and quality of life parameters [10]. It is of note that data from randomized clinical trials or observational open-label studies do not usually take into consideration patients aged $\geq 65$ years, although they represent a consistent and difficult-to-treat subgroup of psoriatic patients. In fact, disease management in elderly patients is often complicated by contraindications and a higher risk of side effects related to the frequent coexistence of comorbidities and previous treatment failures. Clinical data obtained from trials on younger populations should, therefore, not be automatically attributed to elderly patients, because clinical management of Pso and PsA patients aged $\geq 65$ years requires special considerations. Novel biological agents to treat Pso could represent a strategic option for this patient subgroup due to the improved safety profile together with the ability to modulate the biological responses induced by TNF- $\alpha$ [11]. Etanercept, a human TNF- $\alpha$ receptor fused to the Fc portion of human IgG1, is a competitive inhibitor of TNF- $\alpha$ $[5,6]$. Adalimumab is a recombinant human IgG1 monoclonal antibody specific for human TNF $[8,9]$.

The aim of the present retrospective study was to evaluate the long-term efficacy and safety profile of the easyto-use subcutaneous anti-TNF- $\alpha$ agents, etanercept and adalimumab, in the treatment of elderly patients (aged 65 years or more) affected by plaque-type Pso and PsA.

\section{Patients and Methods}

\section{Methods}

The clinical notes of patients referred to the outpatient collaborative Dermatology and Rheumatology Unit of the University of Rome 'Tor Vergata' were revised in order to perform an observational retrospective analysis on patients aged $\geq 65$ years undergoing subcutaneous administration of anti-TNF- $\alpha$ agents, namely etanercept or adalimumab. The tester (A.G.) had no access to demographic data or to the patients. The study protocol was restricted to patients fulfilling the following search criteria: (i) aged $\geq 65$ years, (ii) Pso with/without PsA diagnosis, (iii) received etanercept or adalimumab monotherapy, (iv) recruitment and treatment period from 2005 to 2008 , and (v) treatment duration of at least 6 months.

Diagnosis of PsA was made using CASPAR (Classification Criteria for Psoriatic Arthritis) by an experienced rheumatologist (M.S.C.) [12]. As routine clinical practice, a signed informed consent which specifically allows the publication of clinical data was obtained from all patients before starting the treatment. The study was conducted in accordance with the ethical principles of the Declaration of Helsinki and was consistent with the guidelines for good clinical practice.

Patients affected by PsA undergoing etanercept treatment received $50 \mathrm{mg}$ weekly as a continuous regimen, while patients affected by Pso received $50 \mathrm{mg}$ twice weekly for 12 weeks, followed by $25 \mathrm{mg}$ twice weekly or $50 \mathrm{mg}$ once weekly as a continuous regimen. Adalimumab treatment was given subcutaneously with a first dosage of $80 \mathrm{mg}$ followed by $40 \mathrm{mg}$ every other week as a continuous regimen for patients affected by PsA and Pso.

\section{Patients}

The global elderly patient population affected by PsA and Pso treated in our department included 257 patients. Among these, $173(67.31 \%)$ patients underwent biological treatment. In detail, $89(34.63 \%)$ patients were treated with a monotherapy regimen including $61(23.73 \%)$ patients treated with etanercept and 28 (10.89\%) with adalimumab, representing our study group. 84 patients $(32.68 \%)$ were treated with a biological agent in combination with one or more conventional systemic agents, including 47 (18.28\%) and 37 (14.39\%) patients treated with etanercept and adalimumab, respectively. Another 84 patients were treated with traditional systemic agents, including 38 (14.78\%) patients treated with methotrexate (20 with monotherapy regimen, 18 with combination regimen), 15 (5.83\%) treated with cyclosporine (4 with monotherapy regimen, 11 with combination regimen), 10 (3.89\%) treated with acitretin, $4(1.55 \%)$ treated with Salazopyrin, 3 $(1.16 \%)$ treated with leflunomide, while the remaining 14 patients (5.44\%) were treated with corticosteroids and nonsteroidal antiinflammatory drugs. From this global elderly population a total of 89 unrelated adult patients ( 49 females and 40 males), affected by Pso and PsA and aged between 65 and 82 years (mean age 69.7), fulfilled the search criteria and were included in the study. 61 patients were treated with etanercept (28 females and 33 males) while 28 patients were treated with adalimumab (12 females and 16 males). The study population demographics and baseline characteristics are summarized in table 1 . Previous traditional systemic and biologic treatments with etanercept and adalimumab are summarized in figure 1.

\section{Clinical Parameters}

Clinical and laboratory routine evaluations were performed in our outpatient unit every 3 months during the treatment following routine clinical practice. Patients' evaluation included a general and dermatological physical examination, photographic records, determination of vital signs, evaluation of laboratory results, monitoring of adverse events and evaluation of skin disease activity using the Psoriasis Area and Severity Index (PASI) score. The PASI score is expressed on a scale from 0 to 72 ; assignment of the disease severity takes into consideration the involved surface area, the erythema, the desquamation and the infiltration of psoriatic plaques [13]. PsA activity was evaluated using a visual analogue scale (Pain-VAS) ranging from 0 to 100 indicating the sub- 
Table 1. Demographic characteristics of the study population

\begin{tabular}{lll}
\hline & Adalimumab & Etanercept \\
\hline Patients & 28 & 61 \\
$\quad$ Males & 16 & 33 \\
Females & 12 & 28 \\
Mean age, years & 69.25 & 69.95 \\
$\quad$ Range & $65-75$ & $65-82$ \\
Disease duration, years & 24.12 & 29.2 \\
$\quad$ Range & $4-51$ & $3-64$ \\
Age at onset, years & 44.54 & 40.44 \\
$\quad$ Range & $15-69$ & $7-71$ \\
Mean PASI at BL & 10.40 & 11.33 \\
$\quad$ Range & $0.4-23.8$ & $0.4-68.3$ \\
Mean Pain-VAS & 71.30 & 75.11 \\
$\quad$ Range & $35-100$ & $30-100$ \\
Mean DAS44-ESR & 3.432 & 5.804 \\
$\quad$ Range & $2.44-4.61$ & $4.07-8.25$ \\
\hline
\end{tabular}

$\mathrm{BL}=$ Baseline

jective evaluation of pain of the patient as well as the 44-Joint Disease Activity Score (DAS44-ESR) [14].

Clinical and laboratory data were analyzed using a digital anonymous database and the analysis endpoints chosen were baseline (W0), week 12 (W12), week 24 (W24), week 52 (1 year), 2 years (week 104) and 3 years (week 156). The primary efficacy endpoints were: (i) mean PASI score variation, (ii) proportion of subjects achieving a $\geq 50 \%$ reduction from baseline of PASI score (PASI50) and proportion of subjects achieving a $\geq 75 \%$ reduction from baseline of PASI score (PASI75) at week 12, week 24 and week 52 and thereafter at every time-point ( 2 and 3 years). Treatment efficacy in patients affected by PsA was evaluated using variations of the mean subjective Pain-VAS, as well as the mean DAS44 values. Moreover, treatment adherence was estimated by the proportion of patients continuing therapy compared to baseline (survival rate) at the end of the observation period.

\section{Statistical Analysis}

Continuous variables were presented with mean and standard deviation, categorical variables in absolute frequencies and percentages. Analyses were based on an intent-to-treat with last observation carried forward population.

\section{Results}

The study population comprised in total 89 unrelated consecutive adult patients ( 49 females and 40 males) affected by Pso and PsA: 61 patients treated with etanercept and 28 patients treated with adalimumab. A large proportion of the patients presented concomitant diseases (75.28\%). The main comorbidity associated with

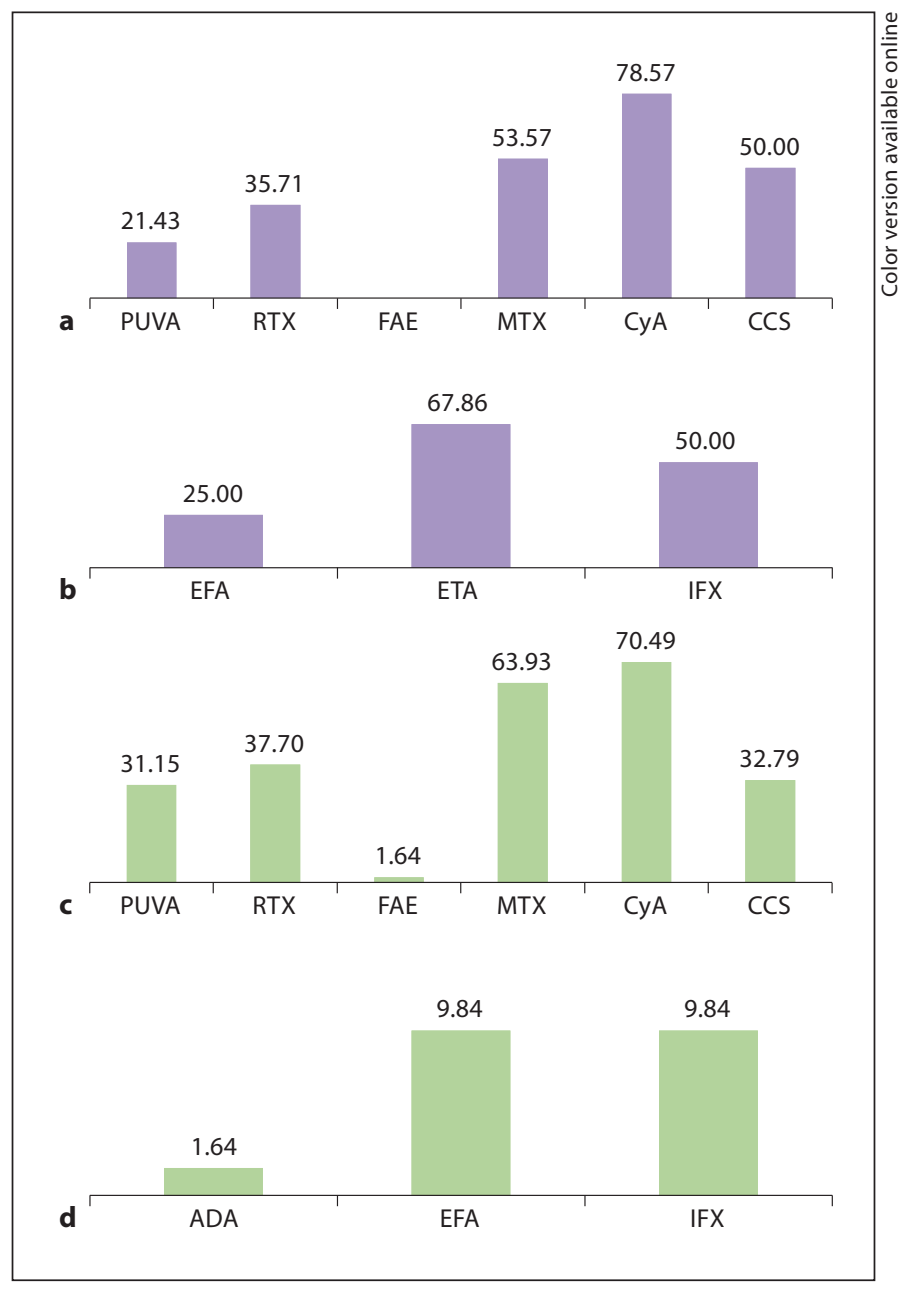

Fig. 1. Percentage of patients who previously received conventional systemic (a, c) and biological (b, d) treatments with adalimumab (a, b) and etanercept (c, d). RTX = Retinoids; FAE $=$ fumaric acid esters; MTX = methotrexate; CyA = cyclosporine; CCS = systemic corticosteroids; EFA = efalizumab; ETA = etanercept; IFX = infliximab; ADA = adalimumab.

plaque Pso was PsA, which was found overall in 62/89 (69.6\%) patients. Cardiovascular comorbidities were also frequent, reported in 49/89 (55.05\%) patients of whom 40 (44.94\%) presented with hypertension and 9 (10.11\%) with a history of acute myocardial infarction. Metabolic abnormalities were also frequent [observed in 31/89 (34.8\%) patients]; type 2 diabetes was most common, found in $25 / 89(28.08 \%)$ patients, while hyperlipemia was frequently present as an associated feature in patients with type 2 diabetes or hypertension and was found in 22/89 (24.7\%) patients. Other renal, infectious 


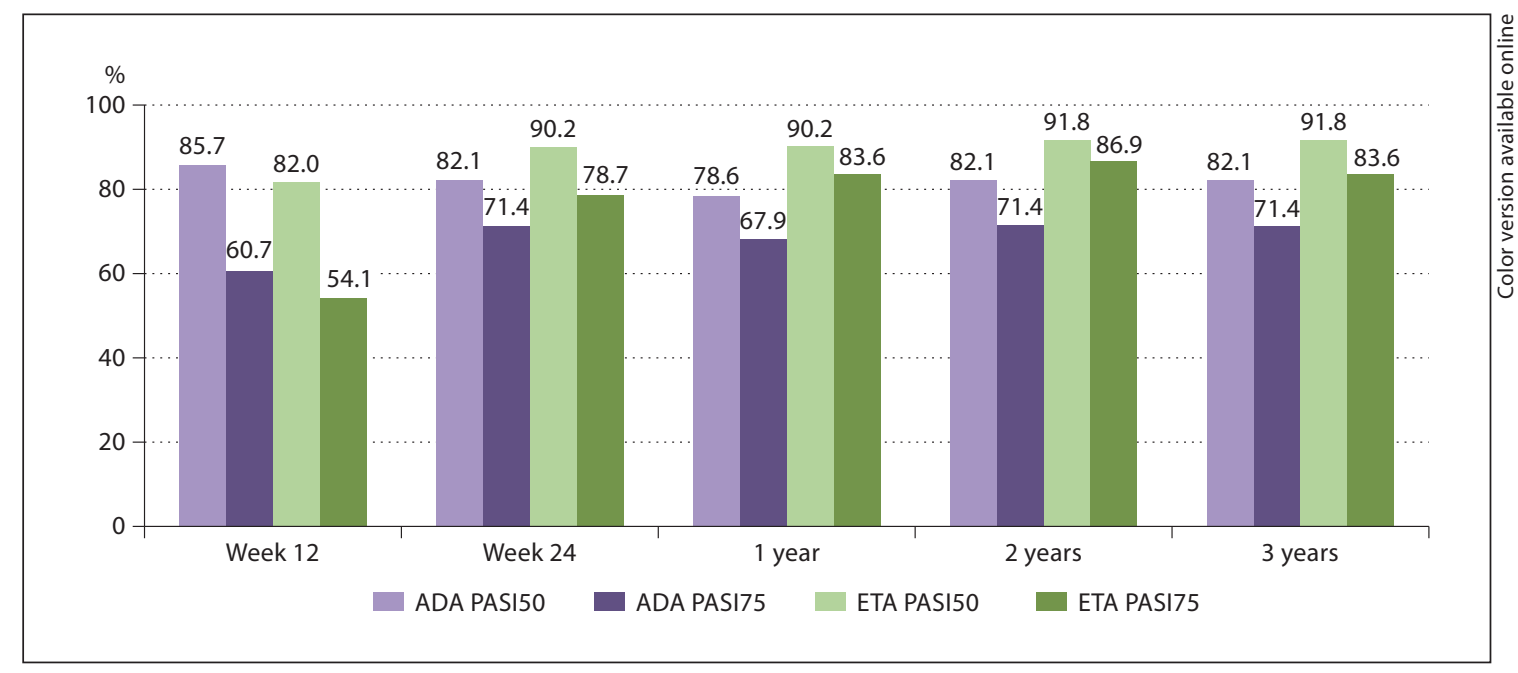

Fig. 2. Etanercept (ETA) and adalimumab (ADA) clinical efficacy as expressed by the PASI50 and PASI75 over the course of the analysis.

Table 2. Comorbidities observed in adalimumab- and etanercepttreated patients

\begin{tabular}{lccc}
\hline Comorbidity & Adalimumab & Etanercept & Total \\
\hline Cardiovascular & $19(67.86)$ & $30(49.18)$ & $49(55.05)$ \\
Metabolic & $10(37.71)$ & $21(34.42)$ & $31(34.83)$ \\
$\begin{array}{l}\text { Chronic renal insufficiency } \\
\text { Infectious }\end{array}$ & $2(7.14)$ & $7(11.47)$ & $7(7.86)$ \\
$\begin{array}{l}\text { Chronic respiratory insuffi- } \\
\quad \text { ciency }\end{array}$ & 0 & $3(4.56)$ & $6(6.74)$ \\
$\begin{array}{l}\text { Others } \\
\quad\end{array}$ & $3(10.71)$ & $3(4.92)$ & $3(3.37)$ \\
\hline
\end{tabular}

Comorbidities were classified in 6 groups: (1) cardiovascular: including mainly hypertension (40 patients) or history of acute myocardial infarction ( 9 patients); (2) metabolic: patients with type II diabetes and hypercholesterolemia; (3) chronic renal insufficiency; (4) infectious: latent tuberculosis (2 patients, etanercept) and chronic hepatitis $C$ virus infection (4 patients, 2 etanercept and 2 adalimumab); (5) chronic respiratory insufficiency: all patients were heavy smokers ( $>15$ cigarettes daily); (6) others, including hyperthyroidism (2 patients, etanercept) and hypothyroidism (4 patients, 1 etanercept and 3 adalimumab).

or respiratory concomitant diseases were also found in a small proportion of the study sample (table 2). Many of these comorbidities were simultaneously present and in particular the number and proportion of patients affected by one, two, three or more than four conditions were 34 (38.2\%), 24 (26.97\%), 6 (6.74\%) and 2 (2.24\%), respectively.

Subcutaneous Anti-TNF- $\alpha$ Treatment in Pso and PsA
Clinical efficacy on skin manifestations in patients treated with etanercept was expressed by a mean PASI score reduction from 11.33 at baseline to 3.02 at week 12, 1.60 at week $24,1.37$ at week 52 (1 year), and 1.31 at week 104 (2 years); it was 1.48 at week 156 (3 years). Mean PASI score variation from baseline was significant $(\mathrm{p}<0.001)$ at every time-point. The proportion of patients achieving a PASI score reduction of $\geq 50 \%$ from baseline at week 12 was $81.97 \%$, increasing to $90.16 \%$ at week 24 and remaining stable at week 52 (1 year), $91.80 \%$ at week 104 ( 2 years) and at week 156 (3 years), while the proportion of patients achieving a PASI score reduction of $\geq 75 \%$ from baseline at week 12 was $54.10 \%$, increasing to $78.69 \%$ at week $24,83.61 \%$ at week 52 ( 1 year), and $86.89 \%$ at week 104 (2 years); it was $83.61 \%$ at week 156 (3 years) (fig. 2). In patients with PsA, after 12 weeks of treatment, clinical assessment demonstrated a consistent improvement in all considered parameters; the mean DAS44-ESR score decreased from 5.80 to 2.29 , and a further reduction of the mean DAS44-ESR score to 1.26 was observed at week 24, and to 0.71 at week 52 (1 year); it was 0.82 at week 104 ( 2 years) and 0.89 at week 156 ( 3 years). The mean PainVAS score decreased from 75.10 to 19.47 at week 12, and a further reduction to 7.23 was observed at week 24 , and to 1.57 at week 52 (1 year); it was 2.63 at week 104 (2 years) and 3.15 at week 156 (3 years). Clinical efficacy on skin manifestations in patients treated with adalimumab was expressed by a mean PASI score reduction from 10.40 at baseline to 2.84 at week 12 and 1.91 at week 24; it was 1.99 


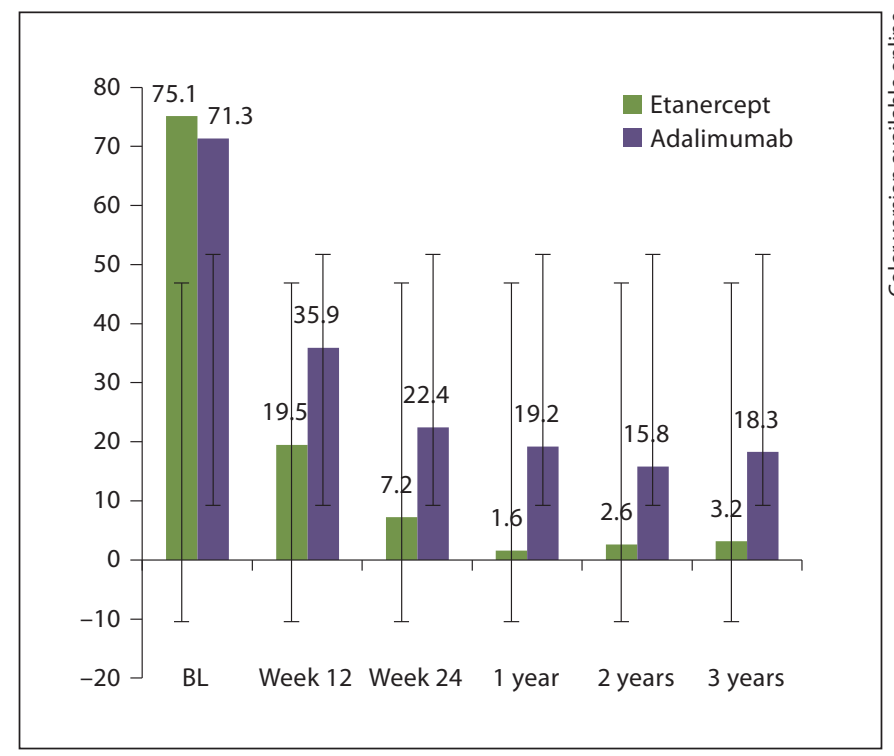

Fig. 3. Mean (SD) Pain-VAS values in etanercept- and adalimumab-treated patients. $\mathrm{BL}=$ Baseline.

at week 52 (1 year), 2.09 at week 104 (2 years), and 1.69 at week 156 (3 years). The mean PASI score variation from baseline was significant $(\mathrm{p}<0.001)$ at every time-point. The proportion of patients achieving a PASI score reduction of $\geq 50 \%$ from baseline was $85.71 \%$ at week 12 , decreasing to $82.14 \%$ at week 24 and to $78.57 \%$ at week 52 (1 year); it was $82.14 \%$ at week 104 (2 years) and remained stable at week 156 (3 years). The proportion of patients achieving a PASI score reduction of $\geq 75 \%$ from baseline was $60.71 \%$ at week 12 , increasing to $71.43 \%$ at week 24 ; it was $67.86 \%$ at week 52 (1 year), $71.43 \%$ at week 104 (2 years), and $71.43 \%$ at week 156 (3 years) (fig. 2). In patients with PsA, assessment after 12 weeks of treatment demonstrated a consistent improvement in all considered parameters; the mean DAS44-ESR score decreased from 3.43 to 2.45 , and a further reduction of the mean DAS44ESR score to 1.88 was observed at week 24, to 1.58 at week 52 (1 year), and to 1.31 at week 104 (2 years); it was 1.44 at week 156 (3 years). The mean Pain-VAS score decreased from 71.30 to 35.91 at week 12 , and a further decrease to 22.43 was observed at week 24 , to 19.22 at week 52 (1 year), and to 15.78 at week 104 (2 years); it was 18.26 at week 156 (3 years). Variations with standard deviations of the mean Pain-VAS and mean DAS44-ESR during the treatment with both agents - etanercept and adalimumab - are displayed in figures 3 and 4 .

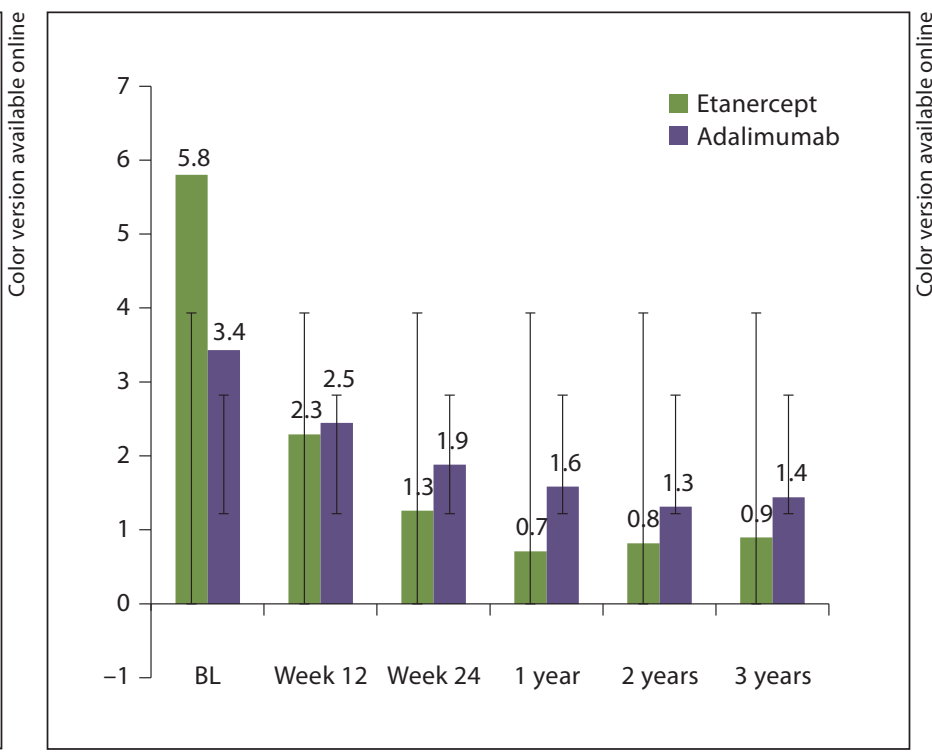

Fig. 4. Mean (SD) DAS44-ESR values in etanercept- and adalimumab-treated patients. $\mathrm{BL}=$ Baseline.

A statistical subanalysis (Student's t test) was performed to investigate if the efficacy profile of etanercepttreated patients differs between patients aged $<75$ (80 subjects) and $>75$ years ( 9 subjects). Results showed no significant differences in all considered efficacy parameters: mean PASI, mean DAS44-ESR, and mean PainVAS $(\mathrm{p}=0.444, \mathrm{p}=0.277, \mathrm{p}=0.303$, respectively, at week 156). The same analysis was not performed for adalimumab-treated patients due to the sample size of patients aged $>75$ years ( 3 subjects).

Treatment adherence was consistently high, expressed by the survival rate at the end of the observation period. Fifteen out of 61 patients (24.59\%) and 11/28 patients (39.28\%) withdrew from the etanercept and adalimumab groups, respectively. The survival rate after 3 years of treatment was 75.40 and $60.71 \%$ for etanercept- and adalimumab-treated patients, respectively. Etanercepttreated patients withdrew due to 2 adverse events ( 15 subjects): a case of repeated tachycardia episodes after the injections and gastric cancer. In 7 cases treatment was interrupted due to loss of efficacy, 4 patients were lost to follow-up, and 2 patients moved to other departments. Adalimumab-treated patients withdrew due to 3 adverse events (11 subjects): a case of severe dyspnea, a case of atrial fibrillation, and a case of severe worsening of a previous glaucoma. In 5 patients treatment was interrupted due to loss of efficacy, 2 patients were lost to follow-up, 
and 1 patient moved to another department. Side effects were rare considering the long-term course of treatment and were mild leading to treatment interruption in 5 cases ( 2 etanercept and 3 adalimumab). Patients treated with etanercept presented with a low titer of antinuclear antibodies (8 cases) as the most common adverse event, but this was not associated with any sign or symptom of autoimmune disease. Other common adverse events were injection site reactions ( 4 cases), weight gain of $\geq 5 \mathrm{~kg}$ ( 4 cases), and mild upper respiratory tract infections (7 cases), of which 2 cases resolved with an antimicrobial specific oral regimen, while the other 5 cases were very mild (tonsillitis or laryngitis) and did not require medical intervention. A case of urinary tract infection requiring antimicrobial-specific treatment and a basal cell carcinoma of the trunk requiring surgical excision were also registered. Two severe adverse events, such as gastric cancer and tachycardia episodes after injection, led to treatment discontinuation. Patients treated with adalimumab presented as the most common adverse event the detection of low-titer antinuclear antibodies (3 cases), which was not associated with any sign or symptom of autoimmune disease, followed by weight gain $\geq 5 \mathrm{~kg}$ ( 2 cases) and dental infection ( 2 cases) resolved with an antimicrobial specific oral regimen and a surgical procedure. Three patients had a very mild upper respiratory tract infection (tonsillitis) which resolved without medical intervention. Other adverse events were urticaria (1 case) and headache (1 case). Only in 3 severe cases - a case of severe dyspnea, a case of atrial fibrillation and a case of severe worsening of a previous glaucoma - was the treatment discontinued.

\section{Discussion}

Despite the availability of various therapeutic options, the long-term management of Pso and PsA with traditional systemic agents is complicated due to treatmentrelated limitations. Particularly in elderly patients the chronic nature of the disease and the frequent association with other major comorbidities or psychological and psychiatric disorders requires efficacious treatment options that can be safely administered on a continuous longterm basis. New biological targeted therapies have changed the paradigm from the traditional intermittent therapy of acute phases of Pso to a continuous long-term control of the disease.

Recent evidence indicates that Pso and PsA are frequently associated with cardiometabolic diseases, which may share the same inflammatory pathway, including

Subcutaneous Anti-TNF- $\alpha$ Treatment in Pso and PsA myocardial infarction, stroke, diabetes, obesity, dyslipidemia and nonalcoholic fatty liver disease. This observation is particularly evident in elderly patients, in whom the identification and prevention of associated risk factors as well as the systemic control of psoriatic inflammation could result in additional improvement of the underlying conditions [15-17]. Moreover, comorbidities should be taken into account when a treatment is proposed, such that a higher risk of side effects and drug interactions between routinely used concomitant medications and traditional therapies for Pso are also considered. Consequently, systemic therapy with agents such as methotrexate, acitretin and cyclosporine should be reserved for short- or mid-term treatment duration in moderate-tosevere Pso cases and should be carefully evaluated in each patient according to their low therapeutic index, possible drug interactions and side effect risk.

In the present study we found that subcutaneous antiTNF- $\alpha$ agents are particularly appropriate for use in elderly patients. Clinical improvements were consistently observed in patients treated with etanercept and adalimumab in all of the analyzed efficacy criteria, indicating an effective and persistent disease control in Pso and PsA patients. From our observations, the mean PASI reduction was significant $(\mathrm{p}<0.001)$ at every time-point compared to baseline and continued to be so throughout the treatment for up to 3 years. Furthermore, the PASI50 and PASI75 percentages demonstrated the reliable skin disease clearance, and the improvements observed in the articular indices (DAS44-ESR and Pain-VAS) demonstrated the long-term stability of the clinical benefits on joint symptoms and inflammation indices.

The analysis was moreover conducted in order to investigate the long-term outcome of etanercept and adalimumab treatment in the presence of different critical conditions, such as advanced age, cardiometabolic diseases, other comorbidities and a history of several previous treatment failures. Considering the long-term treatment duration and the peculiar study population, our analysis confirms the favorable risk/benefit ratio of subcutaneous anti-TNF- $\alpha$ agents.

Elderly patients have a great need for safe treatment, not only of their very often severe skin conditions, but also to prevent possible worsening of comorbidities and their long-term complications. In our analysis the underlying pathological conditions remained stable during the study observation period, and we did not observe progression of the diseases.

Intravenous anti-TNF- $\alpha$ (infliximab) treatments have not been taken into consideration because in our outpa- 
tient collaborative unit we are only able to prescribe subcutaneous anti-TNF- $\alpha$ agents. Intravenous anti-TNF- $\alpha$ (infliximab) is prescribed for patients who attend day hospital or who are hospitalized. In our opinion, home administration of subcutaneous agents represents a practical choice in elderly patients, is usually welcome and avoids infusion-related problems.

Treatment adherence for the long-term period of 3 years was consistently high in both groups with better results in patients undergoing etanercept treatment. Possible reasons for the high adherence rate may be the optimal efficacy and safety profile and the easy administration modality.

Possible limitations of the study are the small patient sample size and the difference among the two groups due to the retrospective nature of the analysis, reporting a casual ratio of approximately $1 / 2$ between the adalimumab and etanercept sample size, respectively. Indeed, this limitation could be a reason for the lesser adherence observed for adalimumab-treated patients, which cannot be explained when considering only the efficacy and safety parameters that were similar to those observed for the etanercept group.

Concerning the safety profile of the two agents administered as continuous monotherapy, we observed no significant differences between etanercept and adalimumab. Tolerability was excellent; we observed only 1 severe adverse event: 1 case of gastric neoplasm leading to etanercept discontinuation. We referred the patient to the Department of Oncology at our university. There it was found that a relationship between etanercept and the neoplasm was not probable in that given case and it was sug- gested not to withdraw the patient from the anti-TNF therapy. We decided to discontinue etanercept anyway and to follow the patient after surgical removal of the neoplasm.

Only few studies have been published on this topic [18], while the use of biological agents in elderly patients with rheumatoid arthritis has been reported in a greater number of publications including a systematic review [19, 20]. This latter review showed that elderly rheumatoid arthritis patients undergoing anti-TNF treatments have a higher number of adverse events and similar efficacy when compared with younger patients.

Although our study did not intend to compare elderly and young patients, we found that our efficacy and safety profile results did not differ from data of major clinical trials, including patients $<65$ years old [4-9].

Complete management of advanced age patients is much needed and in order to achieve optimal compliance and benefit, it is very important to carefully consider quality of life issues, social and financial problems that can negatively affect treatment success and adherence. Treatment goals should be kept simple and individualized for each patient, based on concomitant comorbidities, potential adverse effects, quality of life, drug history, feasibility of follow-up and patient preferences.

\section{Disclosure Statement}

G.B., M.B., A.Z. and R.P. have no conflicts of interest. M.E., A.G., M.S.C. and A.M. served as consultants for Pfizer. S.C. served as a consultant and speaker for Pfizer, Abbott, Schering-Plough, and Centocor.

\section{References}

1 Christophers E, Mrowietz U: Psoriasis; in Freedberg IM, Eisen AZ, Wolff K, Frank Austen K, Goldsmith LA, Katz SI (eds): Fitzpatrick's Dermatology in General Medicine, ed 6. New York, McGraw-Hill, 2003, pp 407427.

2 Naldi L: Epidemiology of psoriasis. Curr Drug Targets Inflamm Allergy 2004;3:121128.

3 Krueger G, Koo J, Lebwohl M, et al: The impact of psoriasis on quality of life; results of a 1998 National Psoriasis Foundation patient-membership survey. Arch Dermatol 2001;137:280-284.

4 Gottlieb AB: Novel immunotherapies for psoriasis: clinical research delivers new hope for patients and scientific advances. J Investig Dermatol Symp Proc 2004;9:79-83.
5 Mease PJ, Goffe BS, Metz J, et al: Etanercept in the treatment of psoriatic arthritis and psoriasis: a randomised trial. Lancet 2000; 356(9227):385-390.

-6 Leonardi CL, Powers JL, Matheson RT, et al: Etanercept psoriasis study group: etanercept as monotherapy in patients with psoriasis. N Engl J Med 2003;349:2014-2022.

7 Chaudhari U, Romano P, Mulcahy LD, et al: Efficacy and safety of infliximab monotherapy for plaque-type psoriasis: a randomized trial. Lancet 2001;357(9271):1842-1847.
8 Papoutsaki M, Chimenti MS, Costanzo A, Talamonti M, Zangrilli A, Giunta A, Bianchi L, Chimenti S: Adalimumab for severe psoriasis and psoriatic arthritis: an open-label study in 30 patients previously treated with other biologics. J Am Acad Dermatol 2007; 57:269-275.

-9 Papoutsaki M, Costanzo A, Chimenti MS, Chimenti S: Adalimumab for the treatment of severe psoriasis and psoriatic arthritis. Expert Opin Biol Ther 2008;8:363-370.

10 Ritchlin CT, Kavanaugh A, Gladman DD, et al; Group for Research and Assessment of Psoriasis and Psoriatic Arthritis (GRAPPA) Treatment recommendations for psoriatic arthritis. Ann Rheum Dis 2009;68:13871394. 
11 Schottelius AJ, Moldawer LL, Dinarello CA, et al: Biology of tumor necrosis factor- $\alpha-$ implications for psoriasis. Exp Dermatol 2004;13:193-222.

12 Taylor WJ, Helliwell PS: Development of diagnostic criteria for psoriatic arthritis: methods and process. Curr Rheumatol Rep 2004;6:299-305.

13 Fredriksson T, Pettersson U: Severe psoriasis - oral therapy with a new retinoid. Dermatologica 1978; 157:238-244.

14 Helliwell PS, Fitzgerald O, Mease PJ: Development of composite measures for psoriatic arthritis: a report from the GRAPPA 2010 Annual Meeting. J Rheumatol 2012;39:398403.
15 Henseler T, Christophers E: Disease concomitance in psoriasis. J Am Acad Dermatol 1995;32:982-986.

16 Gisondi P, Girolomoni G: Cardiometabolic comorbidities and the approach to patients with psoriasis. Actas Dermosifiliogr 2009; 100(suppl 2):14-21.

17 Altobelli E, Petrocelli R, Maccarone M, et al: Risk factors of hypertension, diabetes and obesity in Italian psoriasis patients: a survey on socio-demographic characteristics, smoking habits and alcohol consumption. Eur J Dermatol 2009;19:252-256.
18 Migliore A, Bizzi E, Laganà $\mathrm{B}$, et al: The safety of anti-TNF agents in the elderly. Int J Immunopathol Pharmacol 2009;22:415-426.

19 Genevay S, Finckh A, Ciurea A, et al: Tolerance and effectiveness of anti-tumor necrosis factor alpha therapies in elderly patients with rheumatoid arthritis: a populationbased cohort study. Arthritis Rheum 2007; 57:679-685.

20 Busquets N, Carmona L, Surís X: Systematic review: safety and efficacy of anti-TNF in elderly patients. Rheumatol Clin 2011;7:104112 . 